\title{
Hydroxysafflor Yellow A Promoted Bone Mineralization and Inhibited Bone Resorption Which Reversed Glucocorticoids-Induced Osteoporosis
}

\author{
Li Liu $\left(\mathbb{D},{ }^{1}\right.$ Weiwei Tao $\mathbb{D}^{2},{ }^{2}$ Wenjia Pan, ${ }^{1}$ Li Li $\mathbb{D}^{1},{ }^{1}$ \\ Qiong Yu, ${ }^{1}$ Dawei Zhang, ${ }^{1}$ and Jun Jiang $\mathbb{D}^{3}$ \\ ${ }^{1}$ School of Pharmacy, Guangdong Medical University, Dongguan 523808, China \\ ${ }^{2}$ Center for Translational Systems Biology and Neuroscience, School of Basic Biomedical Science, \\ Nanjing University of Chinese Medicine, Nanjing 210023, China \\ ${ }^{3}$ School of Pharmacy, Jiangsu University, Xuefu Road, Zhenjiang, Jiangsu 212013, China
}

Correspondence should be addressed to Li Liu; 864439037@qq.com and Jun Jiang; jiangjuntcm2007@hotmail.com

Received 17 January 2018; Revised 24 May 2018; Accepted 4 June 2018; Published 5 July 2018

Academic Editor: Adriana Bigi

Copyright (C) $2018 \mathrm{Li}$ Liu et al. This is an open access article distributed under the Creative Commons Attribution License, which permits unrestricted use, distribution, and reproduction in any medium, provided the original work is properly cited.

\begin{abstract}
Glucocorticoids intake is the most common cause of secondary osteoporosis. Clinical studies have shown that $50 \%$ patients develop glucocorticoids-induced osteoporosis (GCIOP) after taking glucocorticoids for more than 6 months. Hydroxysafflor yellow A (HYA) is one main active ingredient in Carthamus tinctorius L. Previous studies have shown that HYA promoted bone marrow mesenchymal stem cells to differentiate into osteoblasts which promoted bone formation. Therefore, we speculated that HYA has a therapeutic effect on GCIOP. However, there is no in vivo evidence about the anti-GCIOP effect of HYA. In this paper, the effect of HYA $(0.1,1.0$, and $10.0 \mu \mathrm{M})$ on bone formation in normal zebrafish was investigated firstly. Secondly, the reversal effect of HYA on GCIOP was also evaluated by zebrafish model. It is demonstrated that HYA not only promoted bone formation in normal zebrafish (compared to Control group), but also reversed glucocorticoid induced bone loss (compared to Prednisolone group) according to the intervention of HYA in upregulating the area of mineralized bones $(p<0.01)$, increasing cumulative optical density $(p<0.01)$, promoting bone formation related gene expression (AKP, Type I, Runx2, OPG, and OCN, $p<0.01$ ), inhibiting bone resorption related gene expression (TRACP, $p<0.01$ ), and elevating whole-body trace mineral elements $(\mathrm{Ca}, \mathrm{P}, \mathrm{K}, \mathrm{Mg}, \mathrm{Zn}$, and $\mathrm{Fe}$ ) levels $(p$ $<0.01$ ). In conclusion, HYA had the potential to prevent and heal GCIOP by promoting bone mineralization, osteoblasts viability, and bone collagen expression and inhibiting bone resorption.
\end{abstract}

\section{Introduction}

Osteoporosis is a disease characterized by microarchitectural deterioration of bone tissue and decreased bone mass, leading to improved bone fragility and the increased risk of fracture [1]. The global population affected by osteoporosis is approximately 100 million, and it is about 69.4 million in China $[2,3]$. Osteoporosis can be divided into primary and secondary type. Glucocorticoids intake is the most common cause of secondary osteoporosis $[4,5]$. Glucocorticoids are very effective at treating human inflammatory and immunosuppressive effects. However, clinical studies have shown that, for patients taking glucocorticoids for more than 6 months,
$50 \%$ develop osteoporosis [6,7]. Furthermore, the incidence of fracture within such groups is approximately $30 \%$ after 5 years of treatment [8]. Thus, osteoporosis is a major limiting factor in the usage of glucocorticoids, even if patients are coprescribed bisphosphonates [9]. Related study has reported that glucocorticoids aggravated the aged osteoporotic status and impairment of skeletal metabolism [3] and thus increase the morbidity of osteoporosis and the risk of osteoporosisrelated fractures. In vivo studies have shown that glucocorticoids treatment causes a decrease in bone formation through the suppression of osteoblasts in mice $[10,11]$. Treatment with the glucocorticoids suppressed the number, activity, and differentiation of osteoblasts during ontogenetic 
TABLE 1: Primer sequences for quantitative real-time PCR.

\begin{tabular}{|c|c|c|}
\hline mRNA & Forward sequence (5' - 3') & Reverse sequence (5' - 3') \\
\hline AKP & CAGGCAAATCAGTGGGAATC & TTGGGCATGTCTGCATCA \\
\hline Type I & CAGGAGCCCAGTGTTGAG & AGCCACCAGACATCTGAGGA \\
\hline Runx2 & GACTCCGACCTCACGACAA & CGTCCCGTCAGGAACATC \\
\hline OPG & CACTGCACAGTCAGGAGGAA & TGCTTTCGATGACGTCTCAC \\
\hline $\mathrm{OCN}$ & GGCGCTACCTGGACCACTG & GCCGTAGAAGCGCCGATAG \\
\hline TRACP & GCСТTCСТTСТTATCTCСТ & СССААТСССТАСАААССТ \\
\hline
\end{tabular}

growth, homeostasis, and regeneration of zebrafish bone [12].

Carthamus tinctorius L. contains flavonoids, chalcones, alkaloids, and other chemical components and is a useful herb in painful osteoporosis [13]. Hydroxysafflor yellow A (HYA) is the main active ingredient of Carthamus tinctorius L. HYA prevented glucocorticoids-induced avascular necrosis of femoral head by inhibiting primary bone marrow mesenchymal stem cells (BMSCs) adipogenic differentiation [14]. In adults, BMSCs differentiate mainly into osteoblasts and adipocytes in the skeleton. Therefore, we firmly believe that HYA could be applied to cure osteoporosis, especially the glucocorticoids-induced osteoporosis (GCIOP).

Many animal models have been successfully used in GCIOP with different times of onset, including rat $[15,16]$, rabbit [17, 18], sheep [19], beagles [20], ewes [21], and pigs [22]. In the last decades, zebrafish has been affirmed as a powerful animal model $[23,24]$ to study bone development. Since the skeletal development of zebrafish has a high degree of similarity to human skeletal development, it is widely used. The zebrafish larvae treated with prednisolone as the model of GCIOP have shown a significant delay in early bone mineralization and regeneration [25].

In present work, we examined the effect of HYA on bone formation in normal zebrafish. And then, the zebrafish larvae were exposed in prednisolone (PNSL) to replicate the GCIOP model which was used to evaluate the therapeutic effects of HYA on GCIOP. Skeleton staining, bone formation/resorption related gene expression, and the bone mineral contents of zebrafish were carried out to assess the anti-GCIOP role of HYA in promoting bone formation and inhibiting bone resorption

\section{Materials and Methods}

2.1. Ethics Statement. Animal experiments were carried out in accordance with the Guide lines for Animal Experimentation of Jiangsu University (Zhenjiang, China) and the protocol was approved by the Animal Ethics Committee of this institution.

2.2. Feeding Zebrafish. Zebrafish embryos were generated by natural mating and reared in 6 well plates containing blank $\mathrm{E}_{3}$ medium $\left(5 \mathrm{mM} \mathrm{NaCl}, 0.17 \mathrm{mM} \mathrm{KCl}, 0.33 \mathrm{mM} \mathrm{CaCl}_{2}\right.$, and $0.33 \mathrm{mM} \mathrm{MgSO}_{4}$ ). Embryos and larvae were maintained on a $14 \mathrm{~h} / 10 \mathrm{~h}$ light/dark cycle and at a water temperature of $28.5^{\circ} \mathrm{C}$ [26].
2.3. Treatment Regime. At 2 days after fertilization (dpf), newly hatched zebrafish larvae were placed into 6 well plates ( $n=15$ larvae in each well) containing blank $\mathrm{E}_{3}$ medium $3 \mathrm{~mL}$. Subsequently, the larvae were divided into different groups including Control (CON, blank $\mathrm{E}_{3}$ medium), prednisolone (PNSL, $25 \mu \mathrm{M}$ ), disodium ethydronate (DE, $15 \mu \mathrm{M}$ DE and 25 $\mu \mathrm{M}$ PNSL), and HYA $(0.1,1.0$, or $10.0 \mu \mathrm{M}$ and $25 \mu \mathrm{M}$ PNSL) on $3 \mathrm{dpf}$. From $6 \mathrm{dpf}$ to $10 \mathrm{dpf}$, larvae were fed with paramecia for $1 \mathrm{~h}$ every day. Following each feeding, the remaining paramecia were washed out and the medium was replaced with blank $\mathrm{E}_{3}$ medium containing drugs or not.

2.4. Skeletal and Matrix Staining. At the $10 \mathrm{dpf}$, all the zebrafish were killed by 3-aminobenzoic acid ethyl ester methanesulfonate (MS-222, $100 \mathrm{mg} / \mathrm{L}$ ). After removal of MS222 solution, zebrafish larvae were fixed in paraformaldehyde solution $4 \%$ in PBS ( $\mathrm{pH} 7.4$ ) and stained with Alizarin Red $\mathrm{S}$ (ARS, $0.5 \% \mathrm{KOH}) .12 \mathrm{~h}$ later, adding fresh prepared bleach with $1.5 \% \mathrm{H}_{2} \mathrm{O}_{2}$ and $1 \% \mathrm{KOH}$ after the staining solution was removed and washed. $1 \mathrm{~h}$ later, all samples were decolored with glycerol and the stained zebrafish was placed under a microscope to observe their stained bones.

2.5. Bone Mineralization Analysis. Quantitative analysis of the area of mineralized bones (AMB) and cumulative optical density (COD) was performed as our previously described $[27,28]$. Images of the ventral aspect of larvae skulls (ARS stained) were acquired using the Inverted fluorescence microscope (Olympus IX71/IX81, Olympus Corporation, Japan). Identical microscopic and camera settings were used for each treatment group. The AMB and COD were calculated using Image J software (National Institutes of Health, Bethesda, MD) for each treatment group $(n=15)$.

2.6. RNA Isolation and Quantitative Real-Time PCR Analysis. Total RNA from larvae were isolated by Trizol (Ambion, Austin, TX), and cDNA was generated by reversetranscription. Expression of AKP, type I collagen, Runx2, OPG, TRACP, and OCN were assessed by quantitative real-time PCR (Rt - PCR). Rt-PCR was performed on an ABI StepOnePlus TM real-time PCR system (one cycle of $95^{\circ} \mathrm{C}$ for $1 \mathrm{~min}$ and $40 \mathrm{cycles}$ of $94^{\circ} \mathrm{C}$ for $10 \mathrm{~s}, 59^{\circ} \mathrm{C}$ for $30 \mathrm{~s}$, followed by melt curve analysis). Relative expression was calculated using the formula $2^{-\Delta \Delta C T}$. Primers used for PCR were listed in Table 1.

2.7. Mineral Contents Detection. The mineral contents in zebrafish larvae were measured using inductively coupled 
plasma-mass spectrometry (ICP-MS) as our previous description [29]. The larvae were treated from $3 \mathrm{dpf}$ to $10 \mathrm{dpf}$ and collected at $10 \mathrm{dpf}$ ( $\mathrm{n}=15$ with three replications). The collected larvae were washed five times in double distilled water and then transferred to centrifuge tubes $(10.0 \mathrm{~mL})$. Immediately, samples were digested with $70 \% \mathrm{HNO}_{3}$ (Tama Chemicals, Kawasaki City, Japan) in a microwave oven for $4 \mathrm{~h}$. Determination of Ca, P, K, Mg, Zn, and Fe was carried out by the $7500 \mathrm{cx}$ ICP/MS system (Agilent Technologies, Santa Clara, CA) equipped with a G3160B I-AS integrated autosampler.

2.8. Statistical Analysis. All data were presented as the mean \pm SD. Differences were analyzed by one-way analysis of variance (Tukey/compare all pairs of columns). Statistical analysis was performed using GraphPad Prism 5 (GraphPad software, USA). Differences with $p<0.05$ were considered significant.

\section{Results}

3.1. HYA Promoted Bone Mineralization in Normal Zebrafish. Bone mineralization is a stage of bone formation. Different concentrations of HYA $(0.1,1.0$, and $10.0 \mu \mathrm{M})$ were incubated with zebrafish in blank $\mathrm{E}_{3}$ medium (Figure 1(a)). Compared with the Control group, HYA significantly increased the area of mineralized bones (AMB, $p<0.01$, Figure $2(a))$ and cumulative optical density (COD, $p<0.01$, Figure $2(\mathrm{~b})$ ). Bone formation related gene expression was examined in our research including AKP, type I collagen, Runx2, OPG, and OCN. HYA promoted the gene expression of AKP, Type I, OPG, and OCN ( $p<0.01$, Figure 3$)$. Meanwhile, HYA inhibited the bone resorption related gene (TRACP) expression $(p<0.01)$. HYA had no significant effect on Runx2 expression. The whole-body $\mathrm{Ca}, \mathrm{P}, \mathrm{K}, \mathrm{Mg}, \mathrm{Zn}$, and Fe levels were improved under the intervention of HYA $(p<0.01$, Figure 4).

3.2. Glucocorticoids Decrease the AMB and COD in Zebrafish. $\mathrm{AMB}$ and COD are important indicators of bone mineralization. In our previous study, Alizarin Red staining was widely used to detect and quantify mineralized bones because it is capable of binding to calcium salts. We examined the optimal concentration of glucocorticoids (Prednisolone and Dexamethasone) in inhibiting bone formation (1.0, 2.5, 10.0, and $25 \mu \mathrm{M})$. Treatment with Prednisolone at $25 \mu \mathrm{M}$ resulted in a significant decrease in AMB and COD compared to controls (Figure 1(b)).

\subsection{HYA Rescued the Inhibition of Bone Mineralization} Caused by Glucocorticoids. HYA has good water solubility. In order to investigate whether HYA is able to attenuate the inhibition of bone mineralization induced by PNSL, three concentrations $(0.1,1.0$, and $10.0 \mu \mathrm{M})$ of HYA were applied from 3 to $10 \mathrm{dpf}$ under $25 \mu \mathrm{M}$ PNSL treatment. Compared to the PNSL-treated group, AMB (Figure 2(a)) and COD (Figure 2(b)) values were significantly increased under the intervention of HYA $(\mathrm{P}<0.01)$. This result indicated that
HYA could rescue the inhibition of bone mineralization in zebrafish.

3.4. HYA Promoted Bone Formation Related Gene Expression and Inhibited Bone Resorption Related Gene Expression. Our results showed that 8 days (from 3 to $10 \mathrm{dpf}$ ) of PNSL treatment significantly reduced the expression of AKP, type I collagen, Runx2, OPG, and OCN ( $p<0.01$, Figure 3$)$. After the treatment of HYA, the expression of these genes was significantly increased compared with the PNSL group $(p<0.01$, Figure 3$)$. On the contrary, HYA inhibited the expression of TRACP, a marker of bone resorption and reversed the elevation of TRACP induced by PNSL $(p<0.01$, Figure 3).

3.5. HYA Promote the Enrichment of the Elements Required for Bone Mineralization. Bone was composed of 69-80 wt.\% calcium phosphate and other components [30]. To confirm the therapeutic effect of HYA on osteogenesis, whole-body $\mathrm{Ca}, \mathrm{P}, \mathrm{K}, \mathrm{Mg}, \mathrm{Zn}$, and Fe contents of larvae were measured by ICP-MS. Compared with the CON, the treatment of PNSL significantly decreased whole-body $\mathrm{Ca}, \mathrm{P}, \mathrm{K}, \mathrm{Mg}, \mathrm{Zn}$, and Fe levels by 2.7-, 2.3-, 20.3-, 6.5-, 8.1-, and 8.8-fold, respectively $(p<0.01)$. After the treatment of HYA, whole-body Ca, P, K, $\mathrm{Mg}, \mathrm{Zn}$, and Fe levels were significantly higher than those in PNSL $(p<0.01$, Figure 4$)$. With the increase of HYA concentration from 0.1 to $10.0 \mu \mathrm{M}$, the contents of these elements in zebrafish also showed an increasing trend and dosedependence.

\section{Discussion}

In the theory of Chinese medicine, Carthamus tinctorius L. is considered to promote blood circulation and remove blood stasis, promote menstruation, and alleviate pain. Recent studies have found that Carthamus tinctorius L. is a useful plant in antiosteoporosis [31]. Carthamus tinctorius L. contains different structural types of compounds including quinochalcones, flavonoids, alkaloids, polyacetylene, aromatic glucosides, and organic acids. Among them, HYA have been reported to be the major active compound with a content more than 13.24 $\mathrm{g} / \mathrm{kg}$ [32]. In vitro studies have shown that HYA promoted the proliferation of human bone marrow-derived mesenchymal stem cells (BMSCs), induced BMSCs osteoblastic differentiation [33], and inhibited BMSCs adipogenic differentiation [14]. However, there is no in vivo study of HYA in antiosteoporosis. In this study, the antiosteoporosis effect of HYA in zebrafish model was first reported.

The zebrafish model is a rapid, high content, in vivo model for glucocorticoids-induced osteoporosis studies. In this study, all of our experiments and data were finished and obtained within 15 days. The HYA used in this paper was isolated from Carthamus tinctorius L. extraction in our laboratory because it is difficult to obtain and high market price. Fortunately, this GCIOP model constructed from zebrafish does not need too much HYA. After calculation, the total amount of HYA used in this study was about $2 \mathrm{mg}$ which is much lower than that of other in vivo organisms, especially rats. 


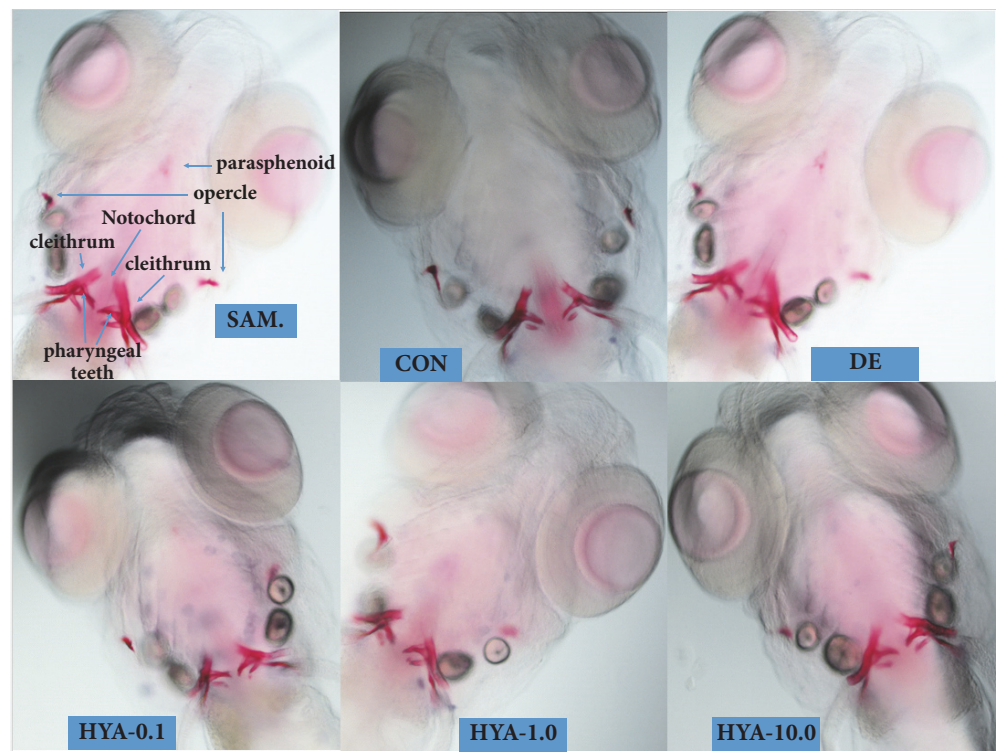

(a)

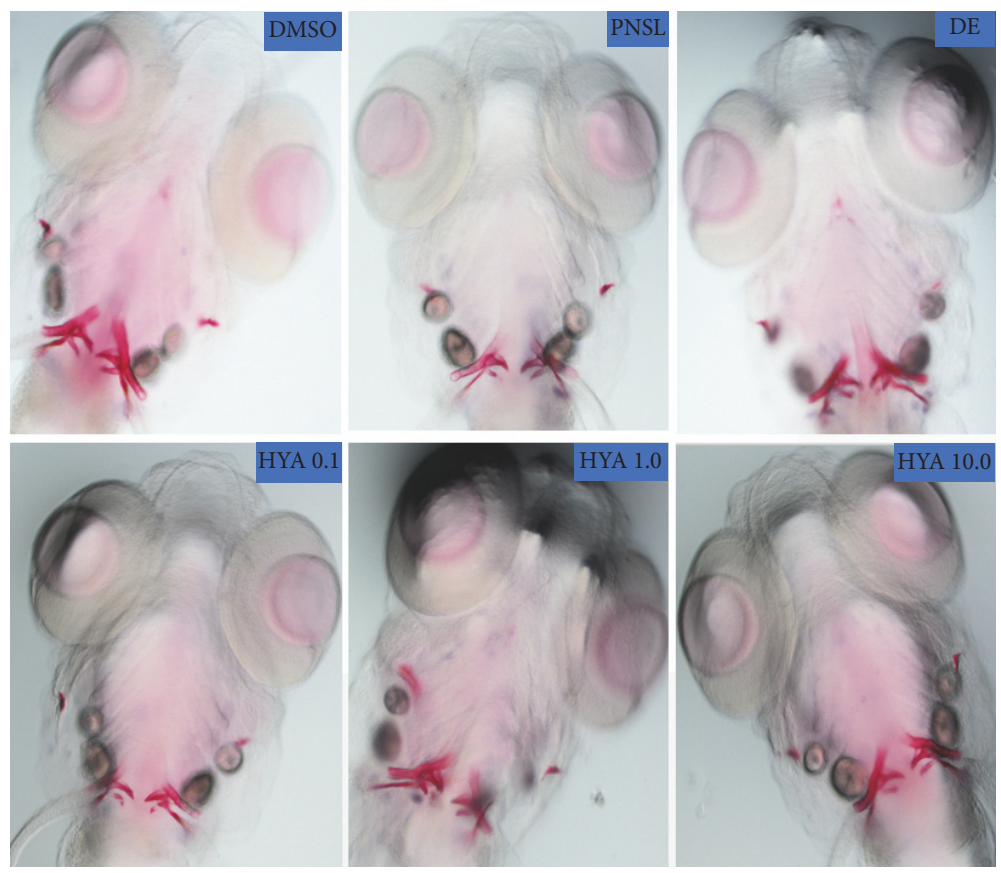

(b)

Figure 1: Ventral view of Alizarin Red stained zebrafish skull at $10 \mathrm{dpf}(\times 100)$. (a) Exposure to blank E3 medium and the effect of HYA on bone mineralization. SAM, the structure of zebrafish bones after staining; CON, blank $\mathrm{E}_{3}$ medium; DE, $15 \mu \mathrm{M}$ disodium ethydronate; HYA 0.1, $0.1 \mu$ M HYA; HYA 1.0, $1.0 \mu$ M HYA; HYA 10.0, 10.0 $\mu$ M HYA. (b) Exposure to prednisolone and the therapeutic effect of HYA on GCIOP. CON, blank $\mathrm{E}_{3}$ medium; DMSO, blank $\mathrm{E}_{3}$ medium + 0.5\% DMSO; PNSL, blank $\mathrm{E}_{3}$ medium + 0.5\% DMSO + $25 \mu \mathrm{M}$ prednisolone; DE, 15 $\mu \mathrm{M}$ disodium ethydronate + PNSL; HYA 0.1, $0.1 \mu \mathrm{M}$ HYA + PNSL; HYA 1.0, 1.0 $\mu \mathrm{M}$ HYA + PNSL; HYA 10.0, 10.0 $\mu \mathrm{M}$ HYA + PNSL. Note: areas of calcified matrix in craniofacial skeleton are stained red.

This model is suitable for different kinds of natural compounds in the antiosteoporosis activity screening. Zebrafish absorbs drugs from the fish water either through the skin or gastrointestinal tract. If the compound is poorly water soluble, dimethyl sulfoxide (DMSO) may be chosen for solubilization. Zebrafish is DMSO tolerant, but the content of DMSO in the medium requires less than $0.5 \%$. The water solubility of HYA is about 0.28 $\mathrm{mg} / \mathrm{mL}$ at $25{ }^{\circ} \mathrm{C}$ [34]. But, prednisolone is a poorly watersoluble compound and DMSO is used for its solubilization. Therefore, in order to remove the interference caused by DMSO and make the administration of HYA more accurate, 


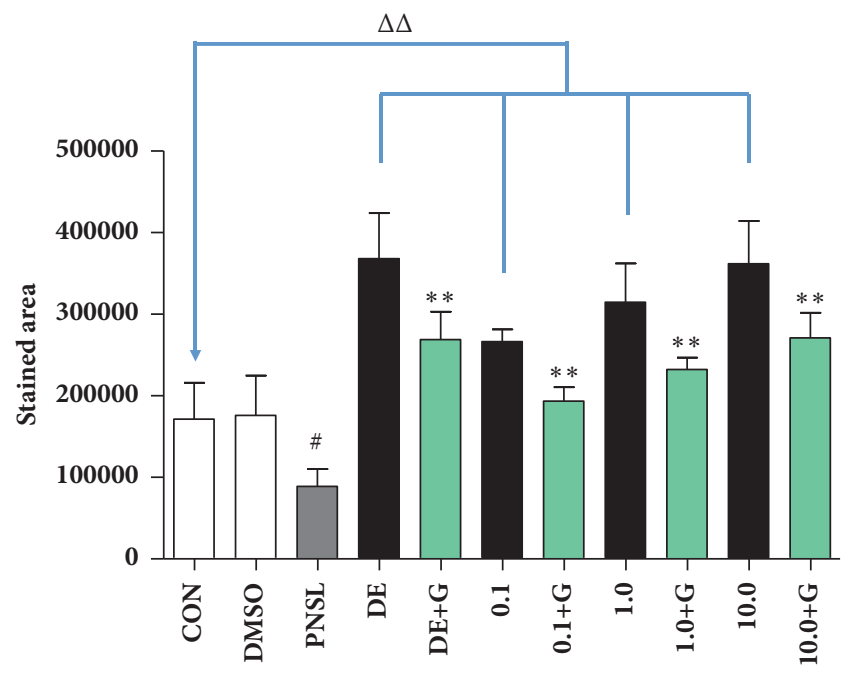

(a)

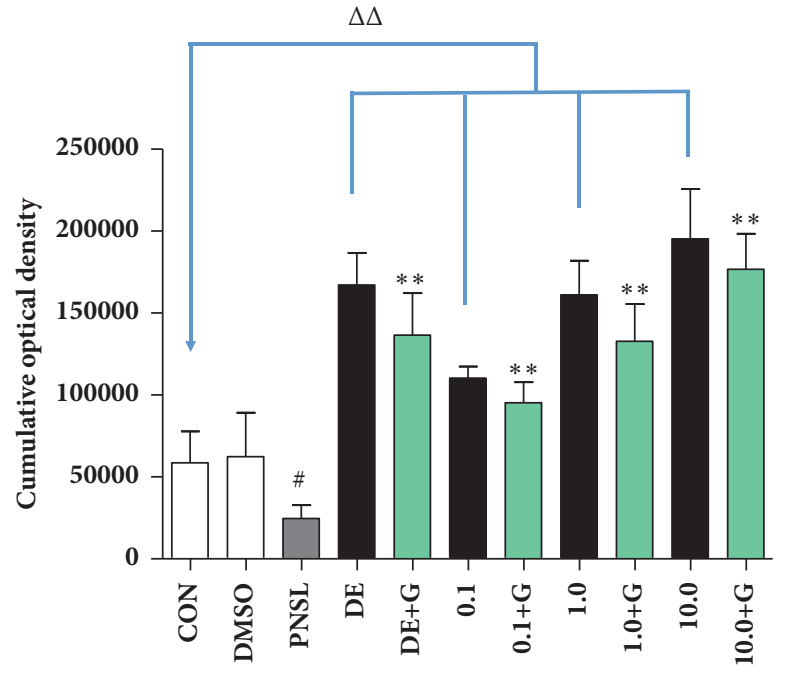

(b)

FIGURE 2: The effect of HYA on mineralization in zebrafish larvae $(n=15)$. (a) Calculation of mineralized area. (b) Calculation of cumulative optical density. Note: CON, blank $\mathrm{E}_{3}$ medium; DMSO, blank $\mathrm{E}_{3}$ medium $+0.5 \%$ DMSO; PNSL, blank $\mathrm{E}_{3}$ medium $+0.5 \% \mathrm{DMSO}+25 \mu \mathrm{M}$ prednisolone; DE, $15 \mu \mathrm{M}$ disodium ethydronate; DE+G, $15 \mu \mathrm{M}$ disodium ethydronate + PNSL; 0.1, $0.1 \mu \mathrm{M}$ HYA; $0.1+\mathbf{G}, 0.1 \mu \mathrm{M}$ HYA + PNSL; 1.0, $1.0 \mu \mathrm{M}$ HYA; 1.0 + G, $1.0 \mu \mathrm{M}$ HYA + PNSL; 10.0, $10.0 \mu \mathrm{M}$ HYA; 10.0 + G, $10.0 \mu \mathrm{M}$ HYA + PNSL. ${ }^{\#}$ Compared with CON, $p<0.01$. ${ }^{\Delta \Delta}$ Compared with CON, $p<0.01$. *Compared with PNSL, $p<0.05 .{ }^{* *}$ Compared with PNSL, $p<0.01$.
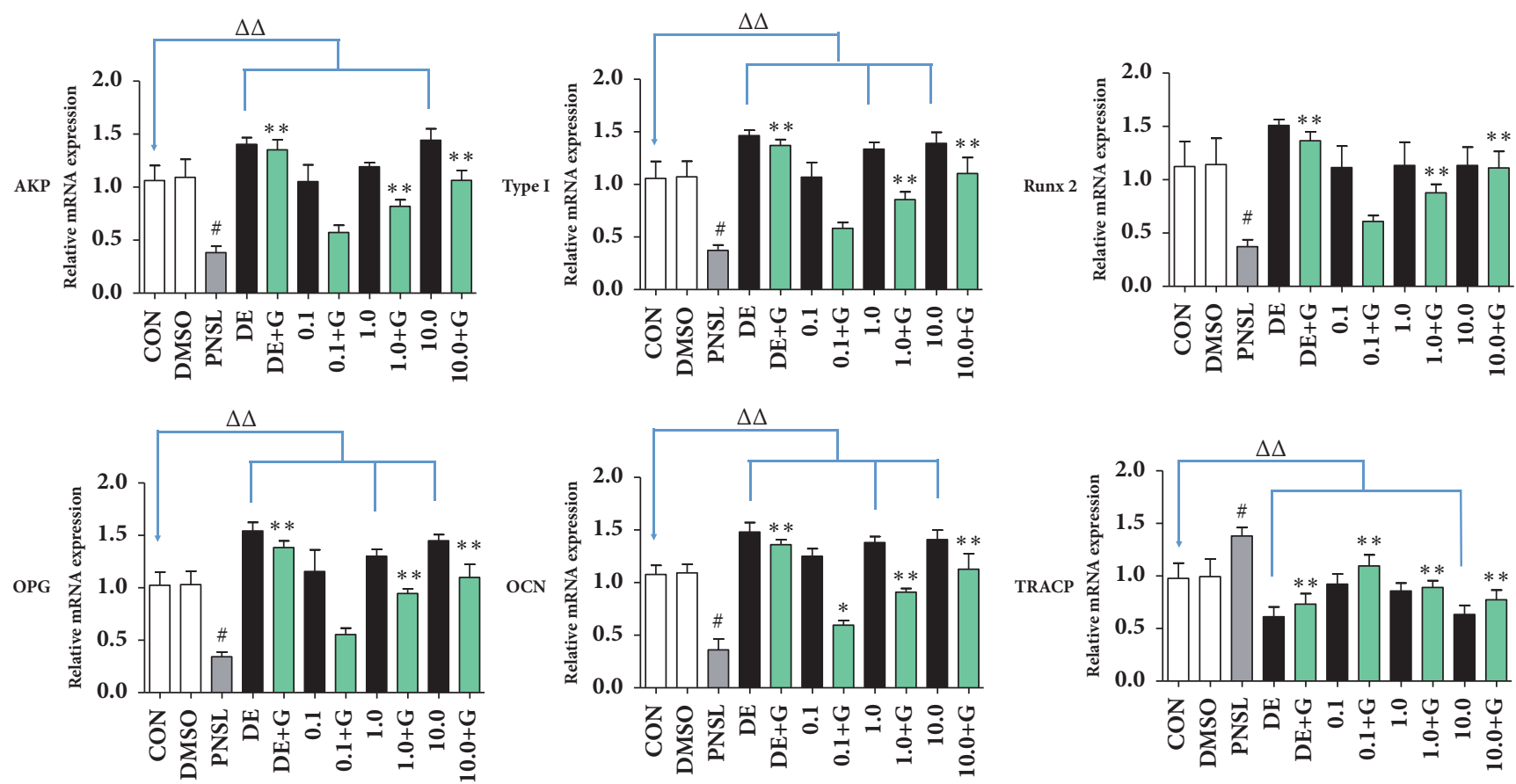

FIGURE 3: Effect of HYA on the expression of related genes in osteoporotic zebrafish larvae $(n=15)$. mRNA levels of the AKP, type I collagen, Runx2, OPG, TRACP, and OCN genes were determined by quantitative real-time PCR. Note: CON, blank $\mathrm{E}_{3}$ medium; DMSO, blank $\mathrm{E}_{3}$ medium + 0.5\% DMSO; PNSL, blank $\mathrm{E}_{3}$ medium $+0.5 \%$ DMSO $+25 \mu \mathrm{M}$ prednisolone; DE, $15 \mu \mathrm{M}$ disodium ethydronate; $\mathbf{D E}+\mathbf{G}, 15 \mu \mathrm{M}$ disodium ethydronate + PNSL; 0.1, $0.1 \mu \mathrm{M}$ HYA; 0.1 + G, $0.1 \mu \mathrm{M}$ HYA + PNSL; 1.0, $1.0 \mu \mathrm{M}$ HYA; 1.0 + G, 1.0 $\mu \mathrm{M}$ HYA + PNSL; 10.0, 10.0 $\mu \mathrm{M}$ HYA; 10.0 + G, $10.0 \mu \mathrm{M}$ HYA + PNSL. ${ }^{\#}$ Compared with CON, $p<0.01 .{ }^{\Delta \Delta}$ Compared with CON, $p<0.01 .{ }^{*}$ Compared with PNSL, $p<0.05$. ${ }^{* *}$ Compared with PNSL, $p<0.01$. 

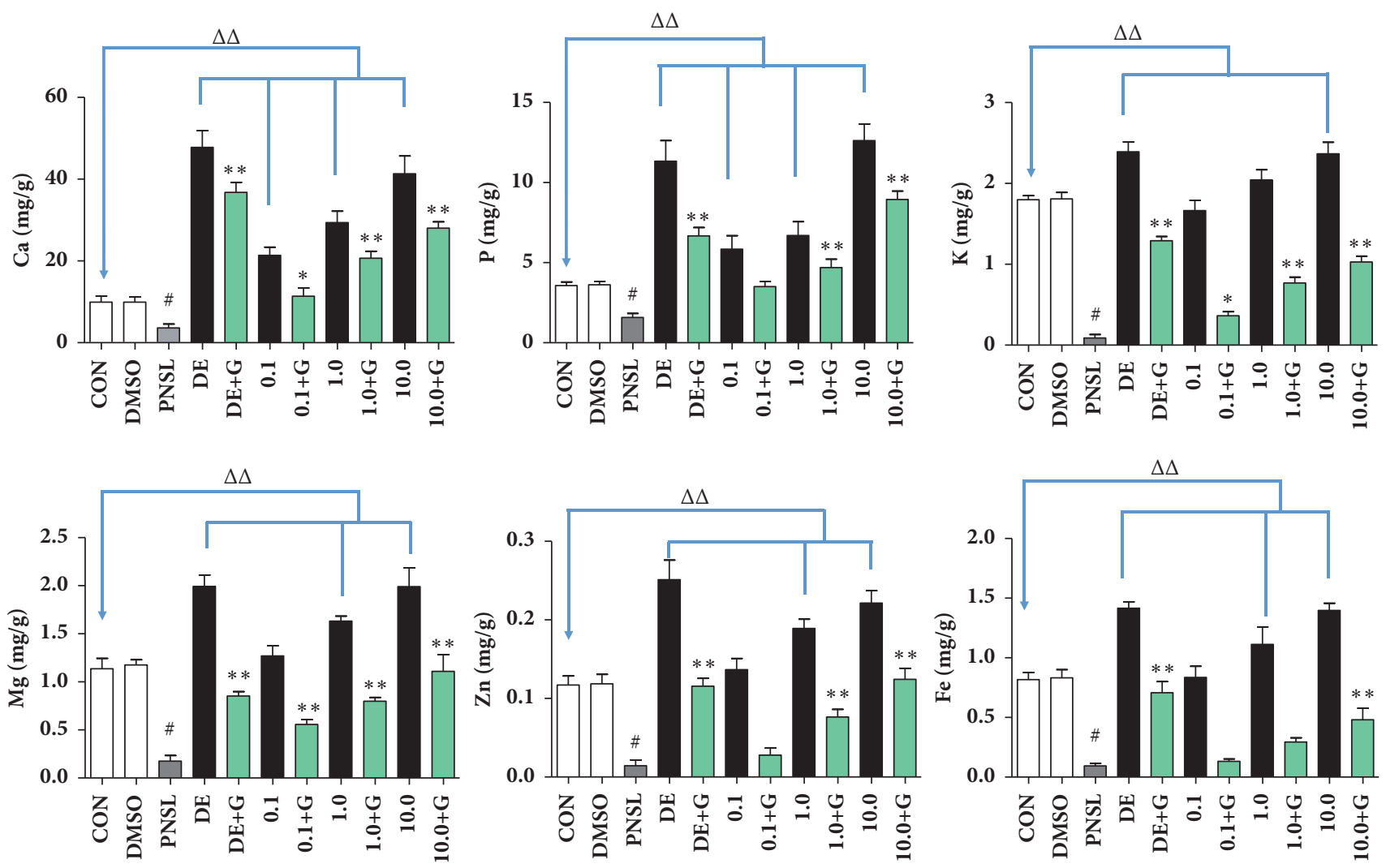

FIGURE 4: Effect of HYA on the mineral contents in osteoporotic zebrafish larvae $(\mathrm{n}=15)$. Note: CON, blank $\mathrm{E}_{3}$ medium; DMSO, blank $\mathrm{E}_{3}$ medium + 0.5\% DMSO; PNSL, blank $\mathrm{E}_{3}$ medium + 0.5\% DMSO $+25 \mu \mathrm{M}$ prednisolone; $\mathrm{DE}, 15 \mu \mathrm{M}$ disodium ethydronate; $\mathbf{D E}+\mathbf{G}, 15 \mu \mathrm{M}$ disodium ethydronate + PNSL; 0.1, $0.1 \mu \mathrm{M}$ HYA; 0.1 + G, $0.1 \mu \mathrm{M}$ HYA + PNSL; 1.0, $1.0 \mu \mathrm{M}$ HYA; 1.0 + G, 1.0 $\mu \mathrm{M}$ HYA + PNSL; 10.0, 10.0 $\mu \mathrm{M}$ HYA; 10.0 + G, $10.0 \mu \mathrm{M}$ HYA + PNSL. ${ }^{\#}$ Compared with CON, $\mathrm{P}<0.01 .{ }^{\Delta \Delta}$ Compared with CON, $\mathrm{P}<0.01 .{ }^{*}$ Compared with PNSL, P $<0.05$. ${ }^{* *}$ Compared with PNSL, $\mathrm{P}<0.01$.

DMSO was also used in this study at the concentration $0.5 \%$.

In the human body, bone is composed of calcium phosphate (69-80 wt. \%, mainly hydroxyapatite), collagen (17-20 wt. \%, mainly type I collagen), and other components (water, minerals, proteins, etc.). The collagen of bone was beneficial to the toughness (energy to fracture) of the bone, mitigating the brittleness of the mineral $\left(\mathrm{Ca}^{2+}, \mathrm{Mg}^{2+}, \mathrm{K}^{+}, \mathrm{Zn}^{2+}, \mathrm{P}\right.$, and others), and contributed to bone strength [13]. The mineral element concentration in zebrafish was in the order of $\mathrm{Ca}^{2+}>$ $\mathrm{P}>\mathrm{K}^{+}>\mathrm{Mg}^{2+}>\mathrm{Fe}^{3+}>\mathrm{Zn}^{2+}$. Treatment of HYA not only promoted the absorption of mineral elements, but also showed a double or triple $(10.0 \mu \mathrm{M})$ concentration of Ca and $\mathrm{P}$ higher than the DMSO group.

According to the literature, excessive $\mathrm{Fe}^{3+}$ exacerbates bone loss [35]. Our previous clinical study (117 postmenopausal women) also elaborated the serum ferritin (Fer) level which was significant negatively correlated with BMD (femoral neck and lumbar spine, $p<0.01$ ). In addition, there was a significant positive correlation between serum Fer levels and serum PINP levels $(p<0.01)$. Serum Fer levels showed a significant positive correlation of serum $\beta$-CTX levels $(p<0.01)$. Therefore, postmenopausal $\mathrm{Fe}^{3+}$ overload exacerbated bone loss by promoting the degradation of type
I collagen. However, all the zebrafish larvae used in this study were younger than $10 \mathrm{dpf}$ which were in a stage where bone formation was greater than bone resorption [36]. Therefore, iron was an essential element involved in zebrafish larvae physiological functions. Glucocorticoids-induced iron concentration significantly decreases in zebrafish; HYA reversed the decrease in iron concentration $(p<0.01)$ and presented a dose-dependent manner from 0.1 to $10.0 \mu \mathrm{M}$. This conclusion was consistent with the area of mineralized bones and cumulative optical density.

AKP was secreted by osteoblasts and promoted bone mineralization. OCN is the dominant noncollagenous protein of the bone matrix. OPG is a decoy receptor that inhibits RANKL activation of osteoclasts, thereby decreasing bone resorption. Runx 2 can directly stimulate the differentiation of bone marrow mesenchymal cells into osteoblasts. Moreover, AKP, OPG, and OCN were important indicators of osteoblasts activity. The expression of Runx2 reflects the ability of bone marrow mesenchymal stem cells to differentiate into osteoblasts. Tartrate resistant acid phosphatase, type $5 \mathrm{~b}$ (TRACP), generally reflected the activity of the osteoclasts and the extent of bone resorption. Therefore, all the selected indicators AKP, OCN, OPG, RANK, Runx2, and TRACP are capable of responding to bone resorption and bone formation activity [37]. 


\section{Data Availability}

The data used to support the findings of this study are available from the corresponding author upon request.

\section{Conflicts of Interest}

All authors had no conflicts of interest regarding the publication of this study.

\section{Acknowledgments}

This work was supported by Young Scientists Fund of the National Natural Science Foundation of China (nos. 81503465 and 81703773), Natural Science Foundation of Guangdong Province (no. 2015A030310263), Natural Science Foundation of Jiangsu Province (no. BK20170560), China Postdoctoral Science Foundation (2016M590424 and 2018T110461), and Jiangsu Postdoctoral Science Foundation (1601184C). All institutional and national guidelines for the care and use of laboratory animals were followed.

\section{References}

[1] E. Kamycheva, T. Goto, and C. A. Camargo, "Celiac disease is associated with reduced bone mineral density and increased FRAX scores in the US National Health and Nutrition Examination Survey," Osteoporosis International, vol. 28, no. 3, pp. 781790, 2017.

[2] G. F. Li, Y. Z. Pan, P. Sirois, K. Li, and Y. J. Xu, "Iron homeostasis in osteoporosis and its clinical implications," Osteoporosis International, vol. 23, no. 10, pp. 2403-2408, 2012.

[3] P. P. Rinne, M. K. Laitinen, T. Huttunen, P. Kannus, and V. M. Mattila, "The incidence and trauma mechanisms of acetabular fractures: A nationwide study in Finland between 1997 and 2014," Injury, vol. 48, no. 10, pp. 2157-2161, 2017.

[4] B. L. Langdahl, "Osteoporosis in premenopausal women," Current Opinion in Rheumatology, vol. 29, no. 4, pp. 410-415, 2017.

[5] L. Buckley, G. Guyatt, and H. A. Fink, "American College of Rheumatology Guideline for the Prevention and Treatment of Glucocorticoid-Induced Osteoporosis," Arthritis Care Research, vol. 69, no. 8, pp. 1094-1110, 2017.

[6] L. Winblad, C. G. Larsen, K. Håkansson, B. Abrahamsen, and C. von Buchwald, "The risk of osteoporosis in oral steroid treatment for nasal polyposis: A systematic review," Rhinology, vol. 55, no. 3, pp. 195-201, 2017.

[7] A. D. Adinoff and J. R. Hollister, "Steroid-induced fractures and bone loss in patients with asthma," The New England Journal of Medicine, vol. 309, no. 5, pp. 265-268, 1983.

[8] D. P. Skoner, "Inhaled corticosteroids: effects on growth and bone health," Annals of Allergy, Asthma \& Immunology, vol. 117, no. 6, pp. 595-600, 2016.

[9] R. S. Weinstein, J.-R. Chen, C. C. Powers et al., "Promotion of osteoclast survival and antagonism of bisphosphonate-induced osteoclast apoptosis by glucocorticoids," The Journal of Clinical Investigation, vol. 109, no. 8, pp. 1041-1048, 2002.

[10] H. Kang, H. Chen, P. Huang et al., "Glucocorticoids impair bone formation of bone marrow stromal stem cells by reciprocally regulating microRNA-34a-5p," Osteoporosis International, vol. 27, no. 4, pp. 1493-1505, 2016.
[11] B. Frenkel, W. White, and J. Tuckermann, "GlucocorticoidInduced osteoporosis," Advances in Experimental Medicine and Biology, vol. 872, pp. 179-215, 2015.

[12] K. Geurtzen, A. Vernet, A. Freidin et al., "Immune Suppressive and Bone Inhibitory Effects of Prednisolone in Growing and Regenerating Zebrafish Tissues," Journal of Bone and Mineral Research, vol. 32, no. 12, pp. 2476-2488, 2017.

[13] J. Asgarpanah and N. Kazemivash, "Phytochemistry, pharmacology and medicinal properties of Carthamus tinctorius L.", Chinese Journal of Integrative Medicine, vol. 19, no. 2, pp. 153159, 2013.

[14] H.-J. Zhu, L.-J. Wang, X.-Q. Wang et al., "Hydroxysafflor yellow A (HYSA) inhibited the proliferation and differentiation of 3T3L1 preadipocytes," Cytotechnology, vol. 67, no. 5, pp. 885-892, 2015.

[15] J. Hu, Z. Mao, S. He et al., "Icariin protects against glucocorticoid induced osteoporosis, increases the expression of the bone enhancer DECl and modulates the PI3K/Akt/GSK3 $\beta / \beta$-catenin integrated signaling pathway," Biochemical Pharmacology, vol. 136, pp. 109-121, 2017.

[16] K. Liu, Y. Jing, W. Zhang et al., "Silencing miR-106b accelerates osteogenesis of mesenchymal stem cells and rescues against glucocorticoid-induced osteoporosis by targeting BMP2," Bone, vol. 97, pp. 130-138, 2017.

[17] Z. Yongtao, W. Kunzheng, Z. Jingjing et al., "Glucocorticoids activate the local renin-angiotensin system in bone: possible mechanism for glucocorticoid-induced osteoporosis," Endocrine Journal, vol. 47, no. 2, pp. 598-608, 2014.

[18] F. Zhou, C. Rong, K. Wang, C.-S. Wang, and Y.-T. Zhang, "Investigation on the role on perindopril for prevention and treatment of glucocorticoid-induced osteoporosis in rabbits," Zhongguo gu shang = China journal of orthopaedics and traumatology, vol. 29, no. 1, pp. 52-57, 2016.

[19] B. B. Kalpakcioglu, K. Engelke, and H. K. Genant, "Advanced imaging assessment of bone fragility in glucocorticoid-induced osteoporosis," Bone, vol. 48, no. 6, pp. 1221-1231, 2011.

[20] L. D. Quarles, "Prednisone-induced osteopenia in beagles: Variable effects mediated by differential suppression of bone formation," American Journal of Physiology-Endocrinology and Metabolism, vol. 263, no. 1, pp. E136-E141, 1992.

[21] P. Chavassieux, A. Buffet, P. Vergnaud, P. Garnero, and P. J. Meunier, "Short-term effects of corticosteroids on trabecular bone remodeling in old ewes," Bone, vol. 20, no. 5, pp. 451-455, 1997.

[22] K. E. Scholz-Ahrens, G. Delling, B. Stampa et al., "Glucocorticosteroid-induced osteoporosis in adult primiparous Göttingen miniature pigs: Effects on bone mineral and mineral metabolism," American Journal of Physiology-Endocrinology and Metabolism, vol. 293, no. 1, pp. E385-E395, 2007.

[23] S. Pasqualetti, T. Congiu, G. Banfi, and M. Mariotti, "Alendronate rescued osteoporotic phenotype in a model of glucocorticoid-induced osteoporosis in adult zebrafish scale," International Journal of Clinical and Experimental Pathology, vol. 96, no. 1, pp. 11-20, 2015.

[24] R. Barrett, C. Chappell, M. Quick, and A. Fleming, "A rapid, high content, in vivo model of glucocorticoid-induced osteoporosis," Biotechnology Journal, vol. 1, no. 6, pp. 651-655, 2006.

[25] E. De Vrieze, M. A. H. J. Van Kessel, H. M. Peters, F. A. T. Spanings, G. Flik, and J. R. Metz, "Prednisolone induces osteoporosis-like phenotype in regenerating zebrafish scales," Osteoporosis International, vol. 25, no. 2, pp. 567-578, 2014 
[26] C. Wynnyckyj, S. Omelon, K. Savage, M. Damani, D. Chachra, and M. D. Grynpas, "A new tool to assess the mechanical properties of bone due to collagen degradation," Bone, vol. 44, no. 5, pp. 840-848, 2009.

[27] B. Chen, G.-F. Li, Y. Shen, X. Huang, and Y.-J. Xu, "Reducing iron accumulation: a potential approach for the prevention and treatment of postmenopausal osteoporosis," Experimental and Therapeutic Medicine, vol. 10, no. 1, pp. 7-11, 2015.

[28] B. Chen, Y.-L. Yan, C. Liu et al., "Therapeutic effect of deferoxamine on iron overload-induced inhibition of osteogenesis in a zebrafish model," Calcified Tissue International, vol. 94, no. 3, pp. 353-360, 2014.

[29] Q. Cheng, X. Zhang, J. Jiang et al., "Postmenopausal Iron Overload Exacerbated Bone Loss by Promoting the Degradation of Type I Collagen," BioMed Research International, vol. 2017, pp. 1-9, 2017.

[30] H. Yin, S. Wang, Y. Zhang, M. Wu, J. Wang, and Y. Ma, “Zuogui Pill improves the dexamethasone-induced osteoporosis progression in zebrafish larvae," Biomedicine \& Pharmacotherapy, vol. 97, pp. 995-999, 2018.

[31] Y. J. Wei, C. M. Wang, X. T. Cai, Y. Zhan, and X. B. Jia, "Establishment of zebrafish osteopenia model induced by dexamethasone," Establishment of zebrafish osteopenia model induced by dexamethasone, vol. 48, no. 2, pp. 255-260, 2013.

[32] Y. Zhan, Y. J. Wei, E. Sun, F. J. Xu, and X. B. Jia, “Twodimensional zebrafish model combined with hyphenated chromatographic techniques for evaluation anti-osteoporosis activity of epimendin A and its metabolite baohuoside I," Yao Xue Xue Bao, vol. 49, no. 6, pp. 932-937, 2014.

[33] J. Jiang, L. Feng, J. Li, E. Sun, S.-M. Ding, and X.-B. Jia, "Multielemental composition of suet oil based on quantification by ultrawave/icp-ms coupled with chemometric analysis," Molecules, vol. 19, no. 4, pp. 4452-4465, 2014.

[34] A. M. Ferreira, P. Gentile, V. Chiono, and G. Ciardelli, "Collagen for bone tissue regeneration,” Acta Biomaterialia, vol. 8, no. 9, pp. 3191-3200, 2012.

[35] B. Hong, Z. Wang, T. Xu, C. Li, and W. Li, "Matrix solidphase dispersion extraction followed by high performance liquid chromatography-diode array detection and ultra performance liquid chromatography-quadrupole-time of flightmass spectrometer method for the determination of the main compounds from Carthamus tinctorius L. (Hong-hua)," Journal of Pharmaceutical and Biomedical Analysis, vol. 107, pp. 464472, 2015.

[36] X. W. Liang, Y. F. Li, and H. Li, "Effects of Hydroxyl Safflor Yellow A on osteogenic differentiation of human bone marrow stromal stem cells," Oral Biomedicine, vol. 8, no. 2, pp. 90-94, 2017.

[37] S. Wang, M. Sun, and Q. Ping, "Enhancing effect of Labrafac Lipophile WL 1349 on oral bioavailability of hydroxysafflor yellow A in rats," International Journal of Pharmaceutics, vol. 358, no. 1-2, pp. 198-204, 2008. 

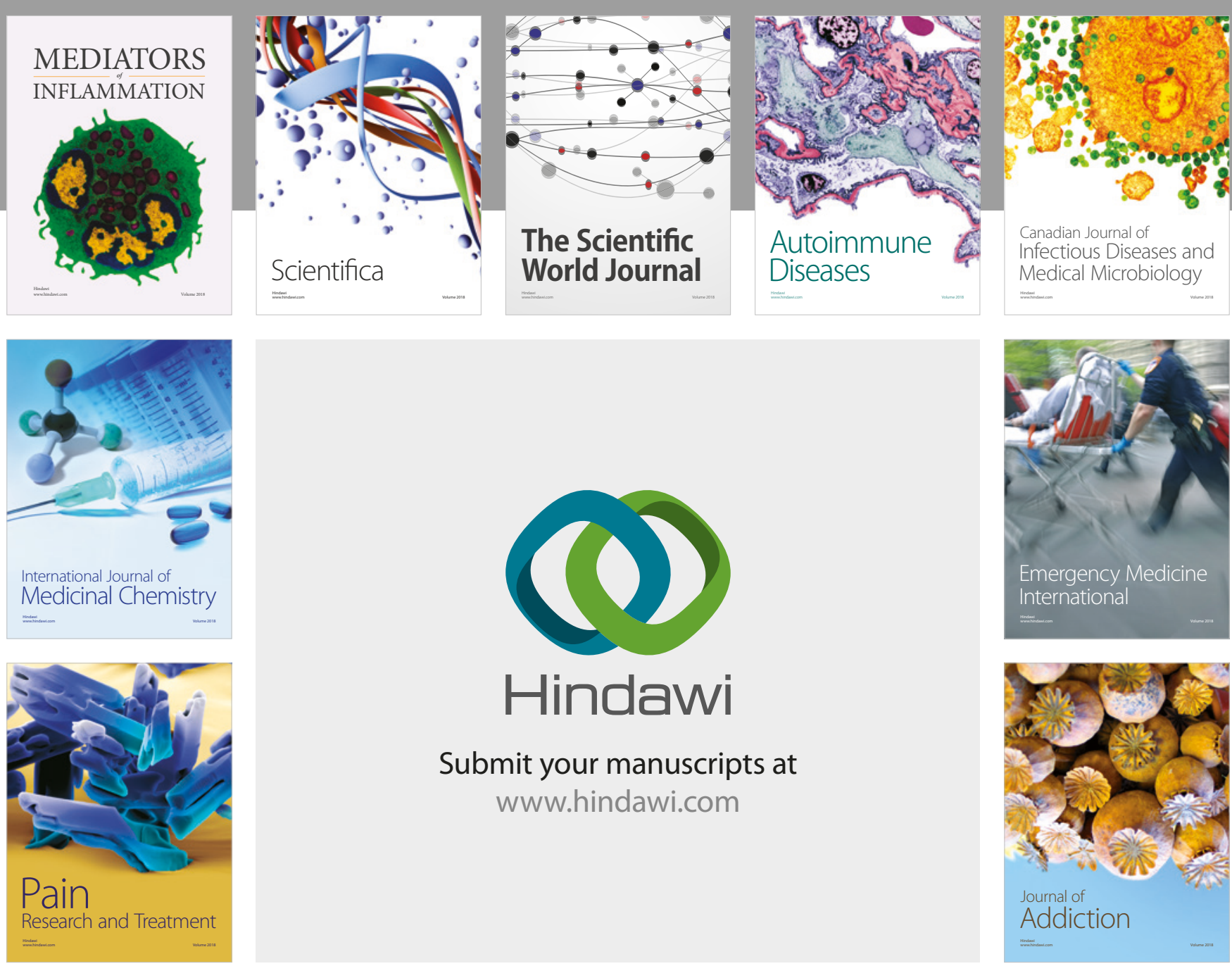

Canadian Journal of
Infectious Diseases and Medical Microbiology

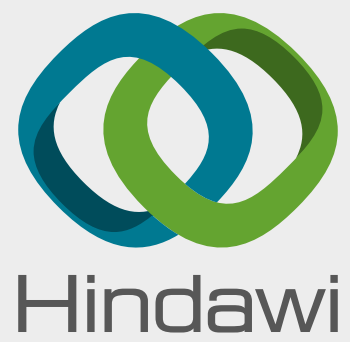

Submit your manuscripts at

www.hindawi.com
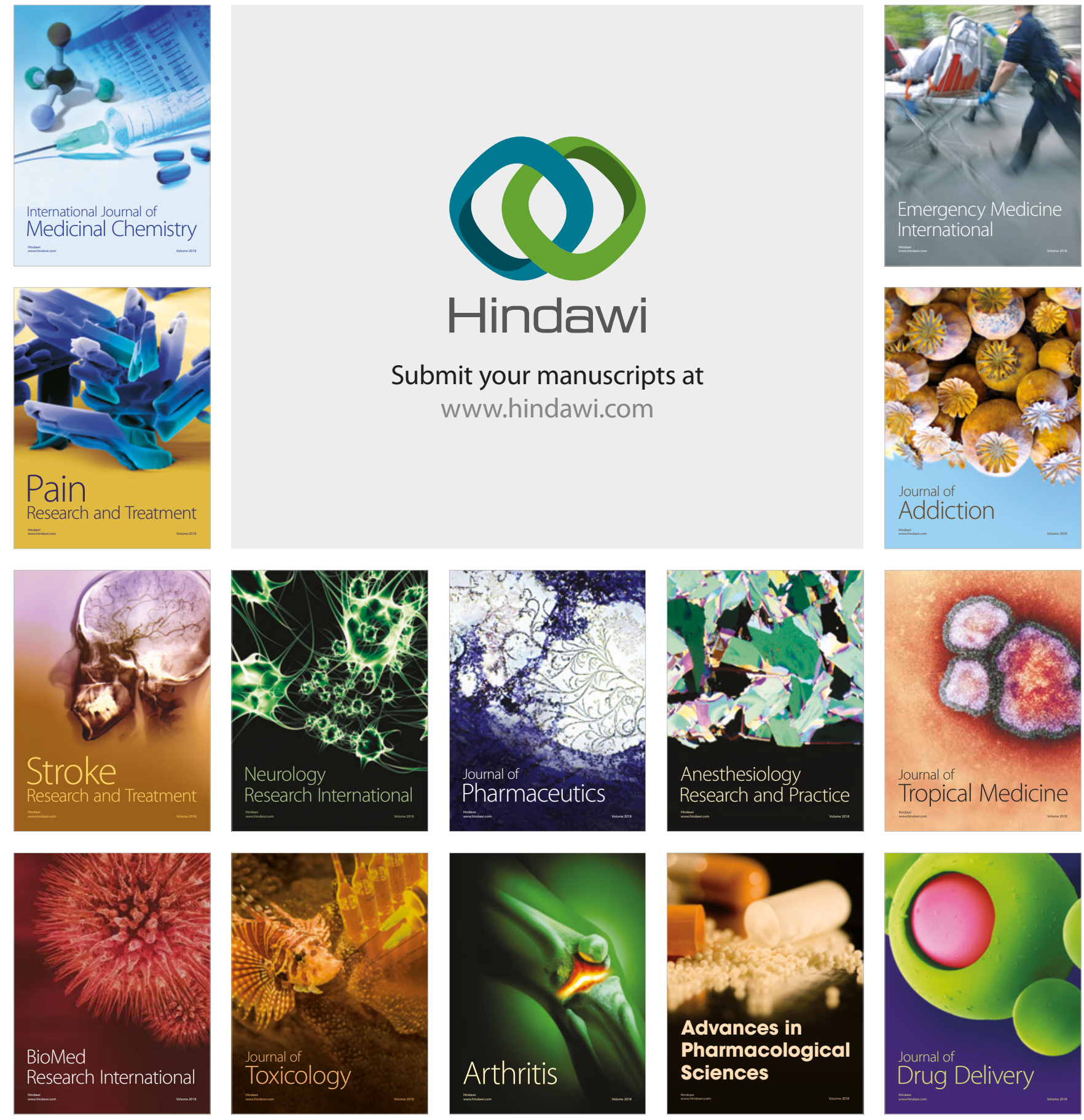